Review

\title{
Inflammatory Bowel Disease and Current Treatment Options in Dogs
}

\author{
Handan Hilal Arslan \\ Department of Internal Medicine, Faculty of Veterinary Medicine, \\ Ondokuz Mayis University, 55139, Atakum, Samsun/Turkey, Turkey
}

\author{
Article history \\ Received: 01-03-2017 \\ Revised: 11-07-2017 \\ Accepted: 19-07-2017 \\ Corresponding Author: \\ Handan Hilal Arslan \\ Department of Internal \\ Medicine, Faculty of Veterinary \\ Medicine, Ondokuz Mayis \\ University, 55139, Atakum, \\ Samsun/Turkey, Turkey \\ E-mail: hharslan@hotmail.com
}

\begin{abstract}
Inflammatory Bowel Disease (IBD) is an idiopathic and chronic gastrointestinal failure of cats, dogs and some farm animals such as horses. Since the long and difficult classical therapy of the disease has various side effects such as malignancy and lymphoma, new therapeutic methods are needed. However, a safe and standardized treatment protocol has not been developed, yet. Therefore, IBD treatment is still a big dilemma for both veterinary and human medicine. In this review, IBD and new possible therapeutic alternatives were evaluated for dogs.
\end{abstract}

Keywords: Inflammatory Bowel Disease, Novel Treatment, Dogs

\section{Introduction}

Inflammatory Bowel Disease (IBD) represents a group of chronic, inflammatory disorders in the gastrointestinal system in animals as well as humans (Haas et al., 2014). It is a progressive disease with poorly characterized etiology (Jackson and Jewell, 2015). IBD is especially important for dogs, because large number of dogs suffer from chronic or recurrent gastrointestinal problems (Cerquetella et al., 2010; Haas et al., 2014).

The 'inflammatory bowel disease' term is applied in veterinary medicine to idiopathic inflammation which is characterized by infiltration of inflammatory cells in the intestinal mucosa and occurs in any area of the gastrointestinal tract (Jergens et al., 1992; Suchodolski et al., 2012). There are different types of diseases defined by affected intestinal region and observed inflammatory cells (German et al., 2003).

The various forms of IBD are classified by anatomic location and the predominant cell type involved in animals. Lymphocytic-plasmacytic enteritis is the predominant form in cats and dogs, followed by eosinophilic inflammation. There are occasional reports of inflammation with a granulomatous pattern (regional enteritis). A neutrophilic predominance in the inflammatory infiltrate is rare. In addition, a mixed pattern of cellular infiltrate is described on many occasions. Certain unique IBD syndromes occur more often in some breeds, such as the protein-losing enteropathy/nephropathy complex in Soft-coated Wheaten Terriers, immunoproliferative enteropathy of
Basenjis, IBD in Norwegian Lundehunds and histiocytic ulcerative colitis in Boxers (Craven et al., 2004; Defarges, 2016).

\section{Pathogenesis}

There are various genetic, environmental and immunoregulatory factors affected etiology and pathogenesis of IBD in dogs as well as humans (Hanauer, 2006; Minamoto et al., 2015).

Routine hematology, parasitic and bacteriologic fecal analyses, dietary trials, radiographic imaging and histopathologic examination of intestinal biopsy specimens provide data to eliminate other causes of chronic enteropathy (Cerquetella et al., 2010).

In dogs, IBD occur a consequence of a deregulation of mucosal immunity in predisposed animals. One of the most studied mechanism to explain development of chronic intestinal inflammation is the loss of tolerance to antigens (food, intestinal bacteria, etc.) that could justify the development of chronic intestinal inflammation. In the dogs with IBD, IgE is increased when compared with healthy dogs. This data could show the immune mediated basis of the disease and hypersensitivity reactions in the pathogenesis. In addition, it could explain the mode of action of immunomodulant drugs in the treatment (Slovak et al., 2014).

Eosinophils and mast cells increase in many dogs with Eosinophilic Gastro-Enteritis (EGE) according to the mucosal barrier failure, independently of the primary cause (bacterial, chemical, etc.) of the inflammation can also lead to further antigen exposure, thereby the chronic 
process becomes and is enforced by decreased apoptosis of lymphocytes, as demonstrated in dogs with IBD when compared to control dogs (Cerquetella et al., 2010).

Genetically predisposition is an important risk factor for IBD. It is believed that IBD is caused by inappropriate and permanent inflammatory response to communal microorganisms in a genetically susceptible host. Recent studies showed the main mechanisms, relationships between the mucosal immunity and the disease, interaction of genetic factors with microbial and environmental reasons and different biological effects in different phenotypes (Khor et al., 2011).

\section{Incidence}

There is no information about incidence for dogs and cats in IBD. Because, characterisation of many cases is still incomplete and detailed histopathological criteria for the differential diagnosis are needed (Hall, 2009).

The peak incidence of IBD in human population was observed between 1960sand 1980s in North America. It was reported that approximately 1000000 people have IBD and about 30000 new cases are defined annually in the U.S.A. (Hanauer, 2006). The incidence is evenly divided between UC and CD. For Europe, IBD incidence is at least three times higher in northern Europe than the southern part (Shivananda et al., 1996).

Epidemiological researches showed that the incidence of IBD has dramatically elevated in the last 50 years. There are more than 160 genetic risk loci, most of them cannot be fully defined. IBD is getting more common especially in developing countries and in migrants populations. These findings also show the importance of environmental influences on the genetic predisposition. Another important predisposition factor is the smoking and CD incidence was reported higher in smokers in the West. However, appendectomy, tonsillectomy, breast feeding and antibiotic use are other indicated factors of high IBD incidence (Siew et al., 2014).

\section{Sex and Age}

There is no apparent age, sex, or breed predisposition associated with IBD in animals; however, it may be more common in German Shepherds, Yorkshire Terriers, Cocker Spaniels and purebred cats. The mean age reported for development of clinical disease is 6.3 year in dogs and 6.9 year in cats, but IBD has been documented in dogs $<2$ years old (Defarges, 2016).

Intestinal inflammation has been reported usually in dogs and cats 6 month sold and much younger. However, it is generally not evaluated as IBD and other reasons, such as Trichomonas infections, are considered the cause of this kind of inflammatory failures. In addition, IBD could be histologically confused with villus lymphoma (Hall, 2009).
More comprehensive information is present for human. Although IBD can be seen any age of individuals, its incidence is higher in young adults. It was reported that IBD is usually seen in people 15-30 years old. Approximately $10 \%$ of IBD cases has been observed in 18 years old. Minimum incidence of UC and $\mathrm{CD}$ is observing in humans $50-70$ years old. $\mathrm{CD}$ is occurring frequently in females (1.8 times higher than males). However, UC is observed almost in equal ratio between females and males. Higher socioeconomic populations are under elevated risk for both diseases (Hanauer, 2006; Khor et al., 2011).

\section{Localisation in Gastrointestinal System (GIS)}

A chronic, continuous or intermittent inflammation is the evident observation of Crohn's Disease (CD) and Ulcerative Colitis (UC) that are considered as IBD in humans. Although $\mathrm{CD}$ affects all parts of the intestinal tract, it generally locates in ileum and colon. Granulomas that could descript an ulcerative and inflammatory disease can involve in the whole intestinal part in CD (Khor et al., 2011). The most common location is the ileocecal region, followed by the terminal ileum alone, diffuse small bowel, or isolated colonic disease in decreasing order of frequency (Hendrickson et al., 2002). However, these signs are mostly restricted in the superficial layers of the colon (mucosa or superficial part of the submucosa) in UC (Khor et al., 2011).

The localisation of IBD is very important for the diagnosis of the disease in humans. Relapses are more occurring in CD patients with ileocolitis than patients with ileitis or colitis alone. For UC, the symptoms and complications are more frequent and serious when colon is involved to the disease. The importance of the anatomical localisation of IBD is not clear in cats and dogs. It was reported that although histological changes may be observed in whole parts of GI tract, IBD frequently affects to the small intestine especially ileum and jejunum (Craven et al., 2004; Suchodolski et al., 2012).

\section{Clinical Signs and Severity}

There is no specific symptom for IBD in dogs. The common symptoms are weight loss, persistent or recurrent vomiting and/or diarrhoea. These findings generally associated with as cites (if hypoalbuminemia is observed) or pallor of mucous membranes (if chronic gastrointestinal bleeding is occurred) (Cerquetella et al., 2010).

Because of IBD is a very individual disease, it could be difficult to make comparison among patients. Numeric data is necessary to objective evaluation of the disease severity. Therefore an "Activity Index" was reported by Jergens et al. (2003) in dogs with IBD. The index useful to the management of clinical patients, in addition initial response to treatment could be evaluate and long term process would be detected (Jergens et al., 2003). 


\section{Canine IBD Activity Index}

"The Canine IBD Activity Index" (CIBDAI) is a simple scoring system. In this system, 6 remarkable gastrointestinal signs were evaluated based on healthy clinical situation of animals and scored $0-3$ by the gastroenterology specialist Table 1. After that the scores are summed, a total cumulative CIBDAI score is obtained and the severity of IBD is evaluated Table 2 (Jergens et al., 2003).

\section{Diagnosis}

For the diagnosis, all possible GI inflammation reasons should have been ruled out (Suchodolski et al., 2012). Beside clinical signs and CIBDAI scores, endoscopy, biopsy and his to pathological investigation are used for the detection of IBD. GI endoscopy is an easy, relatively non-invasive and useful method for determination the active inflammation. The criteria have been developed for qualitative evaluation of endoscopic mucosal appearances Table 3 (Slovak et al., 2014). In addition, his to pathological analysis is another method for the diagnosis of IBD and objective criteria are established in dogs Table 4 (Poullis et al., 2002; McCann et al., 2007; Magro et al., 2013).

However, practical, cheap, sensitive and specific methods are needed for the diagnosis of the disease, still. Blood and faecal markers could fulfil these criteria and may be a good diagnostic alternative. CRP, Tumour Necrosis Factor Alpha (TNF $\alpha$ ) and microalbuminuria have been identified as useful markers of disease activity in human patients with IBD (Poullis et al., 2002; McCann et al., 2007). Nevertheless, McCann et al. (2007) reported that there was no Correlation Between those parameters and clinical symptoms (CIBDAI) and his to pathological findings.

Anti-Neutrophil Cytoplasmic antibody (p-ANCA), Anti-Saccharomyces Cerevisiae (ASCA) were investigated a clinic-pathological diagnostic/monitoring markers in veterinary medicine for IBD. In addition, measurement of mucosal permeability after lactulose andrhamnose administration and $\operatorname{IgG}$ and nitrite concentrations in colonic lavage fluid could be important parameters for the diagnosis and monitoring of the IBD in dogs (Cerquetella et al., 2010).

The serum folate and cobalamin concentrations were also investigated but they were considered as nonspecific findings in IBD. However, these parameters could be important for supplementation during the treatment (Cerquetella et al., 2010).

Recently, Fecal Calprotectin (FC) has been reported as a useful biomarker for evaluation of the intestinal inflammation and IBD in humans. FC is also known as L1 protein, Myoleoid-Related Protein 8/14 (MRP$8 / 14$ ), calgranulin and cystic fibrosis antigen. FC is excreted and stable for 7 days at room temperature in feces and can be measured with a commercially available ELISA immunoassay. FC levels have been found to be significantly elevated in patients with inflammatory and neoplastic conditions (von Roon et al., 2007) and could be a new marker for differential diagnosis of IBD in animals, as well.

\section{Treatment}

The main aim of the treatment of IBD is to depress the inflammation regarding clinical symptoms and histological alterations. Although this approach is beneficial for long-term remission and reduced risks of complications, relapses of the disease are common in many patients. Therefore, recent researches tend to find more effective therapeutic methods to re-install intestinal immune tolerance in IBD (Di Giovangiulio et al., 2015).

Treatments of Canine IBD are generally based on human therapies. Most general and primitive therapeutic procedure in canine and human IBD contains corticosteroids, other anti-inflammatory agents, such as 5-Amino Salicylic Acid (5-ASA) or immunosuppressant in the last decade (Farzaei et al., 2015; Zhang et al., 2016).

Corticosteroids are commonly first choice for the treatment of IBD in dogs. According to retrospective studies, prednisolone is the most used drug alone or in combination. Many studies showed that prednisone has good effects in the treatment of IBD in dogs. But, systemic corticosteroids caused some side effects, such as polyphagia, polyuria, polydipsia, restlessness, panting and behaviour changes in dogs. Long-time usage of corticosteroids can cause obesity, vacuolar hepatopathy, muscle atrophy and weakness, ligament rupture, urinary tract infection, pyoderma and development of diabetes mellitus (Dye et al., 2013). Furthermore, corticosteroids and immunosuppressive drugs combinations caused extra short- and long-term side effects, such as increased liver parameters, allergic reactions, nausea, pancreatitis (Zhang et al., 2016).

The knowledge about treatment alternatives of IBD are changing day by day according to the new experiences. Some researchers reported the malignancy of the therapeutics specifically. For example, immunosuppressive therapies used in IBD may increase the risk of lymphoma (Kotlyar et al., 2015). Pasternak et al. (2013) reported that azathioprine usage was associated with an increased risk of overall cancer in IBD patients, although these data cannot establish causality. In addition, a meta-analysis study demonstrated that the use of thiopurines (azathioprine and 6-mercaptopurine) is associated with a 4-fold increased risk of lymphoma in patients with IBD (Kotlyar et al., 2015). 
Table 1. Evaluation criteria for clinical IBD (Jergens et al., 2003)

\begin{tabular}{|c|c|}
\hline Attitude/activity & 0 normal \\
\hline & 1 Slightly decreased \\
\hline & 2 Moderately decreased \\
\hline & 3 Severely decreased \\
\hline \multirow[t]{4}{*}{ Appetite } & 0 Normal \\
\hline & 1 Slightly decreased \\
\hline & 2 Moderately decreased \\
\hline & 3 Severely decreased \\
\hline \multirow[t]{4}{*}{ Vomiting } & 0 None \\
\hline & 1 Mild (1 time/week) \\
\hline & 2 Moderate (2-3 times/week) \\
\hline & 3 Severe (3 times/week) \\
\hline \multirow[t]{4}{*}{ Stool consistency } & 0 Normal \\
\hline & 1 Slightly soft feces or fecalblood, mucus, or both \\
\hline & 2 Very soft feces \\
\hline & 3 Watery diarrhoea \\
\hline \multirow[t]{4}{*}{ Stool frequency } & 0 Normal \\
\hline & 1 Slightly increased (2-3 times/day) \\
\hline & 2 Moderately increased (4-5 times/day) \\
\hline & 3 Severely increased ( $>5$ times/day) \\
\hline \multirow[t]{4}{*}{ Weight loss } & 0 None \\
\hline & 1 Mild ( $<5 \%$ loss $)$ \\
\hline & 2 Moderate $(5-10 \%$ loss $)$ \\
\hline & 3 Severe $(>10 \%$ loss $)$ \\
\hline
\end{tabular}

Table 2. Means of CIBDAI Scores (Jergens et al., 2003)

\begin{tabular}{llll}
\hline $0-3$ & $4-5$ & $6-8$ & 9 and more \\
\hline Clinically non-significant disease & Mild IBD & Moderate IBD & Intensive IBD \\
\hline
\end{tabular}

Table 3. Qualitative evaluation of endoscopic mucosal appearances (Slovak et al., 2014)

\begin{tabular}{lll}
\hline Appearance & Score & Description \\
\hline Friability & 0 & Absent \\
& 1 & Mild bleeding to touch \\
Granularity & 2 & Marked bleeding to touch \\
& 0 & Normal mucosal texture \\
Erosions & 1 & Mucosal texture increased \\
& 2 & Marked mucosal texture \\
Lymphatic dilatation $^{\text {a }}$ & 0 & Absent \\
& 1 & Only few erosions \\
& 2 & Diffuse erosions \\
& 0 & Absent \\
\end{tabular}

${ }^{\mathrm{a}}$ Defined only during endoscopy

\section{Classical Treatment}

Medical treatment for induction of clinical remission is largely based on anti-inflammatory drugs (corticosteroids) and dietary management (Minamoto et al., 2015). Jergens et al. (2010) carried out the first randomizedcontrolled trial evaluating the efficacy of prednisone versus prednisone and metronidazole in combination in dogs with IBD. The recovery ratio was similar in both treatment groups after 21 days. In addition, both treatment protocols caused significantly decreased mean C-Reactive Protein (CRP) concentrations post-treatment. However, the magnitude of CRP reduction was greatest in dogs receiving prednisone monotherapy. The results of the study showed that oral prednisone immunotherapy was as effective as combined treatment with prednisone and metronidazole in the treatment of canine IBD.

In addition, recent studies focused on the close relationship between the GI microbiota and the host. Because, alterations in the GI microbioata (dysbiosis) has been determined in dogs with chronic GI inflammation as well as humans. This kind of changes and the status of GI microbiota are very important the therapy and the health status. Additionally, it was reported that histolytic ulcerative colitis in dogs has been associated with adherent and invasive Escherichia coli and fluoroquinolone antimicrobial treatment was frequently effective (Minamoto et al., 2015). 
Table 4. Objective criteria for histopathological evaluation of IBD in dogs (McCann et al., 2007)

Epithelium (surface)

Presence of bacteria/fungi

Integrity of epithelium

Goblet cell differentiation

Mononuclear (or other) cell trafficking

Lamina

Haemorrhage, congestion or oedema

Fibrosis

Cellular infiltration

Crypts

Uniformity/symmetry

Deletion

Hypertrophy

Hyperplasia

Goblet cell differentiation

Mononuclear (or other) cell trafficking

Small intestinal villi

Architecture

\author{
None noted +++ \\ Normal or abnormal (if abnormal description \\ of change and severity graded, ++++ ) \\ None noted +++ \\ None noted +++ (and description of cell type) \\ None noted +++ \\ None noted +++ \\ Normal numbers +++ (and description of cell type) \\ Symmetric or asymmetric \\ None noted +++ \\ None noted +++ \\ None noted +++ \\ None noted +++ \\ None noted +++ \\ Normal or abnormal
}

\section{Probiotics}

Microbes in the GI tract have important roles for health in mammalians. Intestinal microbiota provides a defensive barrier against pathogen agents, helps the digestion, stimulates the immune system and gives a nutritional support to enterocytes. Also, dysbiosis, alterations of GI microbes and presence of particular pathogen species (Clostridium perfringens, Salmonella spp., some viruses and parasites) are associated with many GI disorders such as idiopathic IBD in dogs. Therefore, definition of specific microbial community entero types may open up new therapeutic approaches to companion animal health, by designing or prescribing appropriate diets for specific disease phenotypes (Suchodolski et al., 2012).

Many researches have been evaluated harmful effects of microorganisms in GI disorders. However, there is a tendency to investigate helpful effects of beneficial bacteria. Recent studies defined the good effects of probiotics to manipulate the normal intestinal flora (Khor et al., 2011).

Intestinal flora has a regulatory function to the inflammation and this function is probably lost or insufficient in IBD. Researches showed that probiotics may be helpful for restoration the microbial homeostasis in intestines (Khor et al., 2011).

Khor et al. (2011) reported that a probiotic formulation caused a significant increasing of IgA levels in infants and probable enhancement of the intestinal mucosal resistance. Bibiloni et al. (2005) reported that a probiotic preparation (VSL\#3) consisting of a mixture of eight lactic acid bacterial species (Lactobacillus casei, $L$. plantarum, L. acidophilus, L. delbrueckii subsp. bulgaricus, Bifidobacterium longum, B. breve, $B$. infantis, Streptococcus salivarius subsp. thermophilus) is quite effective in maintaining clinical remission in UC. In another study, VSL\#3 and kefir have provided significant recover in the intestinal mucosa of rats with induced IBD (Arslan et al., 2009).

In addition to human and experimental animal studies, although probiotics are used in veterinary medicine (especially in small animal practice) for a long time, there are few data regarding effects of probiotics in dogs. Suchodolski et al. (2010) showed that significant differences of the mucosa-adherent duodenal microbiota were observed between dogs with idiopathic IBD and healthy dogs. Rossi et al. (2014) investigated comparative effects of a commercial product (VSL\#3) that is used in human CD and UC cases and contained different strains of Lactobacillus, Bifidobacterium and Streptococcus and combination of corticosteroid and metronidazol for the treatment IBD in dogs. The results showed that high dose probiotic combination was effective for clinical and histological recovery of IBD and normalization of symbiosis.

Arslan et al. (2012) evaluated therapeutic effects of probiotic bacteria, in comparison with single supportive and symptomatic therapy, in canine parvovirus infection. Only supportive and symptomatic therapy was applied in group I, whereas VSL\#3 was used as adjuvant oral therapy in the group II. The study indicated that probiotics have been shortened the recovery time, under optimal care conditions.

\section{Budesonide}

Budesonide is a non-halogenated glucocorticoid that was developed for IBD in humans. Budesonide has fewer side effects than systemic corticosteriods. In dogs budenisone is used for treatment of allergic dermatitis (topically), chronic inflammatory airway disease (aerosol) and IBD. But there is no controlled studies evaluating the efficacy of oral budesonide treatment for canine IBD (Dye et al., 2013). According to Pietra et al. (2013), budesonide can rapidly absorb and metabolize 
in dogs with IBD. The drug gradually accumulated and it is shown an adequate therapeutic response and no adverse effects occurred.

In another study, 40 dogs were divided into two treatment groups. One group treated with prednisone and second group treated with budenisone. After 3 weeks, complete blood count, biochemical profile and urinalysis were examined. Gastrointestinal endoscopy and biopsies of the intestine with histopathology were performed at Week6.Theresults showed that budisonide is 15 times more potent than prednisolone and may be an effective alternative to prednisone therapy (Dye et al., 2013).

In contrary, Rychlik et al. (2016) reported that budesonide was not effective for treatment of IBD in dogs. The tested drug failed to alleviate clinical symptoms of the disease, decrease the CIBDAI scores or improve the macroscopic appearance of intestinal mucosa. The highest efficacy of budesonide treatment was observed in the histopathological picture of duodenal, jejunal and colonic mucosa.

\section{Mesenchymal Stem Cell Treatment}

Mesenchymal Stem Cells (MSCs) are isolated from various adult tissues and can modulate the immune system. Also, MSCs cause the inhibition of release of pro-inflammatory cytokines (TNF- $\alpha$, Interleukin (IL)-1 $\beta$, IL-6 and interferon) and support the damaged cells, after in-vivo administration (Uccelli et al., 2008).

The application of MSCs is recent concept in treatment of IBD in humans. This technique enhances epithelial proliferation and coordinate re-modelling during the healing process. MSCs have been used in animal model of colitis successfully in pre-clinical studies (González et al., 2009). MSCs have shown immunomodulatory and anti-inflammatory effects in experimental colitis and successful clinical results have been achieved in humans with $\mathrm{CD}$ and UC (Pérez-Merino et al., 2015).

Also, MSCs is one of the current therapies in canine IBD. In veterinary medicine, a recent study of the use of intravenous MSC therapy in spontaneous feline enteropathy was showed a positive and safe clinical response (Pérez-Merino et al., 2015).

In another study, MSCs were obtained from abdominal adipose tissue and used for treatment of IBD in dogs. The study showed that after 2 weeks of therapy, digestive symptoms (vomiting, diarrhoea, soft stools) disappeared and all animals increased activity, appetite and started gaining weight (González et al., 2009).

Thelper-17 (Th17) cells are a group of T helper cells and cause the secretion of cytokines that are members of IL17 family. IL17 cytokines are very important in the inflammation process and promote the production of other pro-inflammatory cytokines, chemokines, hematopoietic growth factors and prostaglandins. Th17 cells have been defined in various inflammatory and autoimmune disorders such as $\mathrm{CD}$, multiple sclerosis, rheumatoid arthritis and psoriasis. Th17 cells consider as responsible for morbidity and mortality of those diseases (Kol et al., 2016).

There are experimental studies regarding determination exact pathways, inhibition of Th 17 polarization and development of potential application protocols to direct therapeutic targets in dogs. The results showed that MSCs may inhibit Th17 polarization in animal models (Kol et al., 2016).

\section{Chondroitin Sulfate and Prebiotics}

Chondroitin Sulfate (CS) is a natural glycosaminoglycan existing in the extracellular matrix and utilized as a substrate by the bacteroid inhabitants of the colon. CS inhibits pro-inflammatory cytokines that producing high levels and characterized by the 'hyper activation' of immune effector cells via their toll-like receptors, particularly macrophages and causing colonic mucosa damage in IBD (Rubinstein et al., 1992; Linares et al., 2015).

Prebiotics are non-digestible food ingredients which support health and helpful for selective stimulation of beneficial bacterial growth (one or limited number of bacteria) in the colon (Geier et al., 2007). Prebiotics could reduce intestinal inflammation, oxidative stress and gut symbiosis (Segarra et al., 2016).

Therefore, there is a new approach with oral CS and prebiotics for the treatment of canine IBD. For instance, Segarra et al. (2016) used a dietary supplement containing CS and prebiotics in dogs with IBD over 180 days. The study showed that long-term oral administration of the combined administration of the supplement with hydrolyzed diet was safe and beneficial for treatment of IBD in dogs.

\section{Polyphenols}

Polyphenols are the secondary plant metabolites which are only present in vegetables and fruits. Apple, bilberry anthocyanin, curcumin, green tea, naringenin, olive oil, pomegranate and ellagic acid, quercetin and resveratrol are regarded as the most commonly used polyphenols (Farzaei et al., 2015).They have antioxidant, anti-inflammatory and immunomodulatory biological effects. In addition, polyphenols have marked ability to modulate inflammation (Biasi et al., 2011). Investigations showed that dietary polyphenols possessed protective properties against various chronic diseases, including cardiovascular and degenerative diseases, diabetes, osteoarthritis and gastrointestinal diseases (Farzaei et al., 2015). In addition, studies on experimental models indicated that polyphones were able to reduced colonic injury (Biasi et al., 2011). Polyphones as dietary supplements can metabolite and help to improving intestinal microbiata and suppressing the inflammatory reactions in the intestinal lumen. Recent years, polyphones have been revealed for the treatment of IBD in humans (Farzaei et al., 2015). 


\section{Infliximab and Biosimilars}

The introduction of biologic therapeutics for treatment of IBD has significantly improved recent years. However, their use is associated with much higher cost compared with traditional treatment options. Therefore, new studies have been carried out regarding cheaper and practical alternatives to biologic therapies. Biosimilars, which are is a bio therapeutic products, similar to the licensed reference product in terms of its quality, safety and efficacy, are getting popular for IBD (Kang et al., 2015).

TNF- $\alpha$ is a pro-inflammatory cytokine and associates many chronic inflammatory autoimmune diseases, such as rheumatoid arthritis, ankylosing spondylitis, psoriatic arthritis, psoriasis, CD and UC (Kang et al., 2015). Infliximab (IFX), a biologic TNF$\alpha$ antagonist, is effective for treating patients with CD and UC (Van de Casteele and Gils, 2015). After the expiration of the patent on IFX, its biosimilars are now available in the market. The first biosimilar of IFX, CTP13 was developed by Celtrion, Inc. (Republic of Korea) and authorized in Europe in September 2013 (Sieczkowska et al., 2016).

CT-P13 were used a case series to indicated the clinical efficacy, safety and inter changeability in the treatment of IBD compared with its originator. As a result, the researchers reported that CT-P13 may have biosimilarity and interchangeability with its originator in IBD (Kang et al., 2015).

\section{Edible Ginger-Derived Nanoparticles}

Ginger, the rhizome of Zingiber officinale, is a widely used natural product. It is consumed as a spice and used as a medicine for the treatment of digestive tract problems like nausea, colic, flatulence, diarrhoea and dyspepsia. In addition, ginger and its active components, including 6-gingerol and 6-shogaol, are anti-oxidative, anti-inflammatory and anti-cancer agents. Recent studies showed that that nanoparticles derived from edible ginger (GDNPs 2) could be a natural and safe alternative for treatment of intestinal inflammations (Zhang et al., 2016).

\section{Conclusion}

IBD is a very important health problem for either human or veterinary medicine. Due to unclear etiopathogenesis and obligatory life-long treatment, the disease seems to a big black hole. In addition, there is a big dilemma, while a life-long treatment is necessary, this classical treatment receipts can cause lifethreatening risks in patients, such as malignancy and lymphoma. Therefore, new, safe, easily accessible and cheap treatment alternatives should be improved for animals as well as humans in IBD.

\section{Funding Information}

There is no any funding to financial support for this study.

\section{Author's Contribution}

Handan Hilal Arslan: Designed, wrote, developed and published the manuscript.

\section{Ethics}

This article is original and contains unpublished material. The corresponding author confirms that all of the other authors have read and approved the manuscript and no ethical issues involved.

\section{References}

Arslan, H.H., D. SaripinarAksu, G. Terzi and C. Nisbet, 2012. Therapeutic effects of probiotic bacteria in parvoviral enteritis in dogs. Rev. Med. Vet., 163: 55-59.

Arslan, H.H., M.Y. Gulbahar, O. Yavuz, A. Bozkurt and G. Terzi, 2009. Effects of differentprobiotics in indomethacin-inducedinflammatory bowel disease in rats. DTW, 116: 11-18.

Biasi, F., M. Astegiano, M. Maina, G. Leonarduzzi and G. Poli, 2011. Polyphenol supplementation as a complementary medicinal approach to treating inflammatory bowel disease. Curr. Med. Chem., 18: 4851-4865.

Bibiloni, R., R.N. Fedorak, G.W. Tannock, K.L. Madsen and P. Gionchetti et al., 2005. VSL\#3 probioticmixture induces remission in patients with active ulcerative colitis. Am. J. Gastroenterol., 100: 1539-1546.

Cerquetella, M., A. Spaterna, F. Laus, B. Tesei and G. Rossi et al., 2010. Inflammatory bowel disease in the dog: Differences and similarities with humans. World J. Gastroenterol., 16: 1050-1056.

Craven, M., J.W. Simpson, A.E. Ridyard and M.L. Chandler, 2004. Canine inflammatory bowel disease: Retrospective analysis of diagnosis and outcome in 80 cases (1995-2002). J. Small Anim. Pract., 45: 336-342.

Defarges, A., 2016. Inflammatory bowel disease in small animals.

Di Giovangiulio, M., S. Verheijden, G. Bosmans, N. Stakenborg and G.E. Boeckxstaens et al., 2015. The neuromodulation of the intestinal immune system and its relevance in inflammatory bowel disease. Front Immunol., 6: 590. DOI: 10.3389/fimmu.2015.00590

Dye, T.L., K.J. Diehl, S.L. Wheeler and D.S. Westfall, 2013. Randomized, controlled trial of budesonide and prednisone for the treatment of idiopathic inflammatory bowel disease in dogs. J. Vet. Intern. Med., 27: 1385-1391. DOI: 10.1111/jvim.12195 
Farzaei, M.H., R. Rahimi and M. Abdollahi, 2015. The role of dietary polyphenols in the management of inflammatory bowel disease. Curr. Pharm. Biotechnol., 16: 196-210.

Geier, M.S., R.N. Butler and G.S. Howarth, 2007. Inflammatory bowel disease: Current insights into pathogenesis and new therapeutic options; probiotics, prebiotics and synbiotics. Int. J. Food Microbiol., 115: 1-11.

German, A.J., E.J. Hall and M.J. Day, 2003. Chronic intestinal inflammation and Haas intestinal disease in dogs. J. Vet. Intern Med., 17: 8-20.

González, M.A., E. Gonzalez-Rey, L. Rico, D. Büscher and M. Delgado, 2009. Adipose-derived mesenchymal stem cells alleviate experimental colitis by inhibiting inflammatory and autoimmune responses. Gastroenterology, 136: 978-989. DOI: 10.1053/j.gastro.2008.11.041

Haas, E., B.C. Rutgen, W. Gerner, B. Richter and A. Tichy et al., 2014. Phenotypic characterization of canine intestinal intraepithelial lymphocytes in dogs with inflammatory bowel disease. J. Vet. Intern Med., 28: 1708-1715. DOI: 10.1111/jvim. 12456

Hall, E.J., 2009. Inflammatory bowel disease in dogs and cats. Hill's GI Booklet.

Hanauer, S.B., 2006. Inflammatory bowel disease: Epidemiology, pathogenesis and therapeutic opportunities. Inflamm. Bowel Dis., 12: S3-9.

Hendrickson, B.A., R. Gokhale and J.H. Cho, 2002. Clinical aspects and pathophysiology of inflammatory bowel disease. Clin. Microbiol. Rev., 15: 79-94. DOI: 10.1128/CMR.15.1

Jackson, M. and D. Jewell, 2015. Metabolomic and clinical disturbances in canine inflammatory bowel disease. FASEB J., 29: 265-267.

Jergens, A.E., F.M. Moore, J.S. Haynes and K.G. Miles, 1992. Idiopathic inflammatory bowel disease in dogs and cats: 84 cases (1987-1990). J. Am. Vet. Med. Assoc., 201: 1603-1608.

Jergens, A.E., C.A. Schreiner, D.E. Frank, Y. Niyo and F.E. Ahrens et al., 2003. A scoring index for disease activity in canine inflammatory bowel disease. Vet. Intern Med., 17: 291-297.

Jergens, A.E., J. Crandell, J.A. Morrison, K. Deitz and M. Pressel et al., 2010. Comparison of oral prednisone and prednisone combined with metronidazole for induction therapy of canine inflammatory bowel disease: A randomizedcontrolled trial. J. Vet. Intern Med., 24: 269-277. DOI: $10.1111 / \mathrm{j} .1939-1676.2009 .0447 . \mathrm{x}$

Kang, Y.S., H.H. Moon, S.E. Lee, Y.J. Lim and H.W. Kang, 2015. Clinical experience of the use of CT-P13, a biosimilar to Infliximab in patients with inflammatory bowel disease: A case series. Dig. Dis. Sci., 60: 951-956. DOI: 10.1007/s10620-014-3392-z

Khor, B., A. Gardet and R.J. Xavier, 2011. Genetics and pathogenesis of inflammatory bowel disease. Nature, 474: 307-317.
Kol, A., N.J. Walker, M. Nordstrom and D.L. Borjesson, 2016. Th17 Pathway as a target for multipotent stromal cell therapy in dogs: Implications for translational research. PLoSOne.

Kotlyar, D.S., J.D. Lewis, L. Beaugerie, A. Tierney and C.M. Brensinger et al., 2015. Risk of lymphoma in patients with inflammatory bowel disease treated with azathioprine and 6-mercaptopurine: A metaanalysis. Clin. Gastroenterol. Hepatol., 13: 847-858. DOI: 10.1016/j.cgh.2014.05.015

Linares, P.M., M. Chaparro, A. Algaba, M. Román and I.M. Arza et al., 2015. Effect of chondroitin sulphate on pro-inflammatory mediators and disease activity in patients with inflammatory bowel disease. Digestion, 92: 203-210. DOI: 10.1159/000439522

Magro, F., C. Langner, A. Driessen, A. Ensari and K. Geboes et al., 2013. European consensus on the histopathology of inflammatory bowel disease. J. Crohn's Colitis, 7: 827-851.

McCann, T.M., A.E. Ridyard, R.W. Else and J.W. Simpson, 2007. Evaluation of disease activity markers in dogs with idiopathic inflammatory bowel disease. J. Small Anim. Pract., 48: 620-625.

Minamoto, Y., C.C. Otoni, S.M. Steelman, O. Buyukleblebici and J.M. Steiner et al., 2015. Alteration of the fecalmicrobiota and serum metabolite profiles in dogs with idiopathic inflammatory bowel disease. Gut Microbes, 6: 33-47. DOI: $10.1080 / 19490976.2014 .997612$

Pasternak, B., H. Svanström, K. Schmiegelow, T. Jess and Ahviid, 2013. Use of azathioprine and the risk of cancer in inflammatory bowel disease. Am. J. Epidemiol., 177: 1296-1305. DOI: 10.1093/aje/kws375

Pérez-Merino, E.M., J.M. Usón-Casaús, C. Zaragoza-Bayle, J. Duque-Carrasco and L. Mariñas-Pardo et al., 2015 Safety and efficacy of allogeneic adipose tissue-derived mesenchymal stem cells for treatment of dogs with inflammatory bowel disease: Clinical and laboratory outcomes. Vet. J., 206: 385-390. DOI: 10.1016/j.tvj1.2015.08.003

Pietra, M., F. Fracassi, A. Diana, T. Gazzotti and G. Bettini et al., 2013. Plasma concentrations and therapeutic effects of budesonide in dogs with inflammatory bowel disease. Am. J. Vet. Res., 74: 78-83. DOI: 10.2460/ajvr.74.1.78

Poullis, A., R. Foster, T.C. Northfield and M.A. Mendall, 2002. Review article: Faecal markers in the assessment of activity in inflammatory bowel disease. Aliment Pharmacol. Ther., 16: 675-681.

Rossi, G., G. Pengo, M. Caldin, A.P. Piccionello and J.M. Steiner et al., 2014. Comparison of microbiological, histological and immunomodulatory parameters in response to treatment with either combination therapy with prednisone and metronidazole or probiotic VSL\#3 strains in dogs with idiopathic inflammatory bowel disease. PLoSOne. 
Rubinstein, A., D. Nakar and A. Sintov, 1992. Chondroitin sulfate: A potential biodegradable carrier for colon-specific drug delivery. Int. J. Pharm., 84: 141-150.

Rychlik, A., A. Kołodziejska-Sawerska, M. Nowicki and M. Szweda, 2016. Clinical, endoscopic and histopathological evaluation of the efficacy of budesonide in the treatment of inflammatory bowel disease in dogs. Pol. J. Vet. Sci., 19: 159-164. DOI: $10.1515 /$ pjvs-2016-0020

Segarra, S., S. Martínez-Subiela, M. Cerdà-Cuéllar, D. Martínez-Puig and A. Muñoz-Prieto et al., 2016. Oral chondroitin sulphate and prebiotics for the treatment of canine inflammatory bowel disease: A randomized, controlled clinical trial. BMC Vet. Res., 10: 12-49. DOI: 10.1186/s12917-016-0676-x

Shivananda, S., J. Lennard-Jones, R. Logan, N. Fear and A. Price et al., 1996. Incidence of inflammatory bowel disease across Europe: Is there a difference between north and south? Results of the European Collaborative Study on Inflammatory Bowel Disease (EC-IBD). Gut, 39: 690-697.

Sieczkowska, J., D. Jarzębicka, M. Meglicka, G. Oracz and J. Kierkus, 2016. Experience with biosimilar infliximab (CT-P13) in paediatric patients with inflammatory bowel diseases. Therap. Adv. Gastroenterol., 9: 729-735.

Siew, C.N., W. Tang, R.W. Leong, M. Chen and Y. Ko et al., 2014. Environmental risk factors in inflammatory bowel disease: A population-based casecontrol study in Asia-Pacific. Gut, 64: 1063-1071. DOI: $10.1136 /$ gutjnl-2014-307410
Slovak, J.E., C. Wang, Y. Sun, C. Otoni and J. Morrison et al., 2014. Development and validation of an endoscopic activity score for canine inflammatory bowel disease. Vet. J., 203: 290-295. DOI: $10.1016 /$ j.tvj1.2014.12.030

Suchodolski, J.S., P.G. Xenoulis, C.G. Paddock, J.M. Steiner and A.E. Jergens, 2010. Molecular analysis of the bacterial microbiota in duodenal biopsies from dogs with idiopathic inflammatory bowel disease. Vet. Microbiol., 142: 394-400. DOI: $10.1016 /$ j.vetmic.2009.11.002

Suchodolski, J.S., M.E. Markel, J.F. Garcia-Mazcorro, S. Unterer and R.M. Heilmann et al., 2012. The fecal microbiome in dogs with acute diarrhea and idiopathic inflammatory bowel disease. PLoSOne, 7: e51907-e51907. DOI: 10.1371/journal.pone.0051907

Uccelli, A., L. Moretta and V. Pistoia, 2008. Mesenchymal stem cells in health and disease. Nat. Rev. Immunol., 8: 726-736.

Van de Casteele, N. and A. Gils, 2015. Pharmacokinetics of anti-TNF monoclonal antibodies in inflammatory bowel disease: Adding value to current practice. J. Clin. Pharmacol., 55: 39-50. DOI: 10.1002/jcph.374

von Roon, A.C., L. Karamountzos, S. Purkayastha, G.E. Reese and A.W. Darzi et al., 2007. Diagnostic precision of fecalcalprotectin for inflammatory bowel disease and colorectal malignancy. Am. J. Gastroenterol., 102: 803-813.

Zhang, M., E. Viennois, M. Prasad, Y. Zhang and L. Wang et al., 2016. Edible ginger-derived nanoparticles: A novel therapeutic approach for the prevention and treatment of inflammatory bowel disease and colitis-associated cancer. Biomaterials, 101: 321-40. 